\title{
OBSERVATIONS ON SURGICAL CASES.
}

\author{
By James Macqueen, F.R.C.V.S., Royal Veterinary College, \\ London.
}

DEFECTIVE teeth.- Imperfect mastication and quidding in horses, when not due to wound, abscess, foreign body such as wire, nail, or wood lodged in the mouth, or to tetanus, is caused generally by some defect of the molar teeth. Sharp edged, irregular, prominent, or "overgrown" molars are common enough, but carious teeth are comparatively rare. The causation of defective teeth is perhaps less important than interesting, but it is usual to ascribe irregularities of the molar tables to unequal development of the jaws. Faulty development may not satisfactorily explain the occurrence on the grinding surfaces of a series of responding pits and projections an inch or more in depth or height, but it supplies a convenient answer to a difficult question. Other explanations have been given, but at best they are speculative and unconvincing.

In some horses, owing probably to unequal length of the jaws, the incisor tables do not respond exactly to each other. Cases of overshot or undershot incisors, though varying greatly in degree, are nearly always associated with the formation of prominences more or less large on the first and sixth molar teeth. In overshot mouth a portion of the tables of the first upper and sixth lower molars escapes wear and projects, and in undershot mouth the unworn part occurs on the first lower and sixth upper molars. The prominences of the first molars seldom cause incon. venience, but their existence in any case of slow or imperfect mastication ought to suggest careful examination of the other teeth. Unworn or protuberant parts of the sixth molars of the lower jaw may be found sloping backwards or inwards, or standing upright and partly imbedded in the palate or gum.

At first the molar tables are fairly level; later, in consequence of wear and, to some extent, of the form of the surfaces of the maxillary joints, the tables become oblique. One effect of the inclination of the grinding surfaces is to sharpen the inner edge of the lower and the outer edge of the upper teeth. If the lower molar arch is too narrow, or the upper arch too wide, inclination of the tables increases, and in time the crowns cross like shears blades. Shear teeth usually preserve their full length, and it is customary to refer to them incorrectly as "overgrown." In the horse gradual elevation of the molar teeth is a natural process. As wear advances at the crown the implanted portion loses volume, and the alveolar cavity shrinks as the tooth ascends. Shear teeth have been less worn at their tables than other teeth, but they are not overgrown. After extraction of a molar the tooth opposed to the gap in the table is no longer worn or restrained by direct pressure. It rises in its socket a little faster than the adjoining teeth, but it does not increase in bulk. If not cut or extracted this tooth eventually bruises the gum or palate, or enters the vacant alveolus and impedes mastication.

Frequently in aged cart horses, and occasionally in horses under five years, imperfect mastication or quidding, with emaciation, may be traced to the formation of spaces between the crowns of the molar 
teeth. Food particles fill the spaces, and sink between the teeth and gum. Caries may attack the crown, but oftener some product of bacterial action in the retained food irritates the alveolar lining, causing periostitis and abscess. Some of the most intractable dental cases have been connected with the lodgment of food between the molar crowns. The disease process advances slowly, and early extraction of the loosened teeth may prevent farther extension. In neglected cases, or in those complicated by abscess and necrosis, the jaw enlarges, pus tracts run from the suppurating alveolus through the expanded bone to a secondary or gravitation abscess, which forms in the submaxillary space, and alveolar fistula may prevent recovery. It should be stated that although the teeth in these cases are loose, extraction may be difficult in consequence of enlargement of the fangs by tuberous growths.

As a preliminary to treatment the patient should be carefully examined, and examination should not be restricted to the teeth. This warning cannot be too rigidly observed when dealing with old horses, in which defects of teeth may co-exist with a disease which makes slaughter preferable to any operation. In dental as in other cases prevention should be better than cure, but preventive treatment is generally impracticable when not impossible. The horse's molars are not often amenable to the art of hygiene which occasionally prescribes measures that cannot be successfully applied. Curative treatment, if less worthy, is more popular than preventive treatment, and, in tooth troubles, far more efficacious. Dentistsequine and canine - are gaining the "Crest of the wave," which sooner or later must wash them into remunerative havens. But they should see to their appliances, which, too often, are both cumbersome and costly. Unlike scalpels and other instruments of a nicer sort, tools for teeth do not readily wear out; and it is not improbable that a few of the heavier variety now used have been handed down from one generation to another. Tooth instruments of alarming size-too large for convenient use--are far too common, but, with no pretence to prophesy, the hope may be entertained that the day is not distant when oldfashioned dental implements will be allowed to rust in their cases, and files of various shapes, hack saws, and circular saws mounted on arms like those of the dentist's drilling machine, will displace the clumsy rasps and clumsier shears. Then, instead of extracting carious teeth, which, by the way, are often fairly sound, cavities will be stopped with the best amalgam. But too much must not be expected from posterity. We must hasten slowly, and at least for a time content ourselves with the tools we have got. Sharp molar edges can be rounded with the rasp, and small projections can be removed by the chisel or by the odontriteur. For prominences of the last molars the chisel can be used, but not without risk to the gum or palate, and if the protuberance is thick the force employed may displace the tooth. Failure to cut, at the first blow, a projection from the sixth upper molar may result in fracture of the alveolar wall and sinking of the tooth into the maxillary sinus. When a similar accident happens in attempting to cut the last lower molar, the result is less serious, probably because the displaced tooth remains concealed. Tooth shears cannot always be used successfully in these cases. Sometimes, owing to insufficient space or to the form or direction of 
the prominence, the shears cannot be set across its length. By chance rather than by skilful guidance the protuberance may be cut, but oftener the shears slip off after merely pinching the prominence, which may be too narrow for space between the closed blades. Occasionally perseverance has been rewarded by oblique fracture extending to the fang, and although the offending projection was replaced by a pit it is doubtful which condition gave more trouble. For shortening teeth that protrude beyond the level of the table, and for cutting shear teeth, Thompson's shears are very good, though sometimes a tooth is loosened as well as cut and extraction must follow sooner or later. Fracture in a bad direction and displacement, accidents which occasionally qualify the action of shears on a projecting tooth, can be avoided by employing a saw. One of the best for the purpose and the least expensive is the American hack-saw extended in a narrow frame. When using this saw, which is very brittle, care should be taken not to jerk or bend it, and to prevent abrasion of the cheek a smooth-cdged plate of zinc should be inserted between the cheek and teetll. How far the hacksaw will be able to assist operators on protuberant tceth cannot be stated positively before further trial. It will cut a molar in a few minutes, but it cannot be used on the sixth molar because of insufficient space behind the tooth to work the saw.

Attempts have been made to save carious molars by scraping and cleansing, and afterwards filling the cavities with various tooth substitutes, on lines similar to those pursued by dentists in the treatment of human teeth, but the relief given lacked permanency. The dentist's drill was not thought of ; had it been used in preparing the cavities for the stopping, the result might have justified continuance of a branch of practice which has been too much neglected by veterinary surgeons. When extraction must be performed, forceps should be tried in all cases where the crown of the tooth can be securely grasped. Santy's forceps will draw any ordinary molar which is not chisel-crowned or brittle from the ravages of caries. The fulcrum supplied with the forceps concentrates too much pressure on a single tooth, which may subside during operation. A short rasp or chisel forms a safer fulcrum. In operating strength is useful if not all important, and art is helpful, but the main thing is the application of the forceps to the tooth - the grasp must be secure. Teeth which have been crushed or fractured at the crown by forceps, and some carious teeth, can only be removed by expulsion or punching out. The upper molars can be reached without difficulty in old horses by trephining the wall of the facial sinus; but in young horses the root of the sixth molar lies too near the orbital case to permit of safe punching. When this tooth must be thrust out a bent punch should be used. The first five lower molars can be punched out after trephining the horizontal border of the maxilla, but the sixth lower tooth, owing to varying direction or the distance of its root from the border, cannot be expelled without risk of damage to the jaw.

Compared with extraction by forceps, expulsion is less difficult, especially in old horses, and decidedly more painful in either old or young. Besides, the healing process is very slow and seldom satisfactory. The opening necessary to operation on the lower teeth can be cleansed and drained, and, perhaps, healed within reasonable time, but the breach made by the punch in the wall of 
an upper tooth socket exposes the facial sinus to infection, and it must be confessed that contamination very frequently occurs. Food gains access to the sinus, and, until the alveolus becomes closed, the first trephine hole, or a second, which may have been made for better drainage, must be kept open. When two upper molars have been punched out the probability of complete recovery is exceedingly doubtful. The gap in the gum diminishes, but the opening leading from the sinus into the alveolus remains in spite of daily attention. In some cases, after constant treatment extending over many months, a medium-sized probe may be passed readily through the partly filled alveolus from the mouth to the sinus. Veterinary surgeons, of more or less experience, sometimes regard the expulsion of molar teeth as a mere surgical bagatelle, and the subsequent treatment as of little consequence. But reports of rapid and complete healing after the punching operation should only be accepted with respectful reserve. Extraction by forceps of an upper molar leaves the bony wall of the alveolus intact, but, in punching, the roof or cap of the alveolus is partly removed. In the first instance food may, and generally does, accumulate in the empty socket without appreciable inconvenience to the horse, and in the second some of the food gains the interior of the sinus. To shut off communication between mouth and sinus the alveolus must be closed at the gum, at the sinus, or between these limits. To expect rapid closure is absurd, and to expect perfect obliteration of the passage, before a very long while, is somewhat akin to the expectation of the man who sought to convert a tin-can into a solid by repeatedly coating its inside with paint. Enthusiasm "is cheap today," indeed, it bids fair to supersede the truth. Equine dental surgery has its disabilities, and to indicate some of these is the purport of the foregoing observations.

\section{EDITORIALARTICLES.}

\section{THE STAMPING OUT OF RABIES}

THE recent marked reduction in the number of cases of rabies must be disappointing to the numerous false prophets who foretold that the efforts of the Board of Agriculture to stamp out the disease would end in failure. It is true we have probably not yet seen the last case, but it is now impossible to doubt that a continuance of the existing regulations will before long be crowned with complete success. The number of cases of rabies in the dog reported in 1896 was 438 , as against 154 in 1897 , and the numbers for the first eleven weeks of 1897 and I 898 were respectively 44 and 6 . It is well, however, to remember that this is not the first occasion on which rabies has been brought to the verge of extermination. In 1889,3 I 2 cases of rabies in the dog were returned, and for the following three years the figures were 129 , 79 , and 38 , respectively. This marked reduction in the number of cases was traceable to the enforcement of muzzling regulations in 